\title{
Specificity of changes in cerebral blood flow in patients with frontal lobe dementia
} Sergio E Starkstein, Ricardo Migliorelli, Alejandra Tesón, Liliana Sabe, Silvia Vázquez,
Martín Turjanski, Robert G Robinson, Ramón Leiguarda
Department of Behavioural Neurology

S E Starkstein

R Migliorelli

A Tesón

A Sabe

Department of Nuclear Medicine

S E Starkstein

$S$ Vázquez

$M$ Turjansk

$\mathrm{R}$ Leiguarda

Department of Clinical Neurology

Raúl Carrea Institute of Neurological

Research, Buenos

Aires, Argentina

S E Starkstein

R Leiguarda

Department of

Psychiatry, University

of Iowa, Iowa City,

Iowa 52242, USA

$R$ G Robinson

Correspondence to:

Dr Sergio E Starkstein

Department of Behavioura

Department of Behaviour

Neurology, Raul Carrea

Institute of Neurological

Research, Ayacucho
1112 Buenos Aires,

1112 Buenos

Argentina.

and in final revised form

2 September 1993.

Accepted 23 September 1993

\begin{abstract}
Eight patients with a clinical diagnosis of probable Alzheimer's disease, eight patients with the clinical diagnosis of frontal lobe dementia, and eight controls were examined with single photon emission tomography (SPECT) using ${ }^{99} \mathrm{Tc}-$ HMPAO. Patients with Alzheimer's disease and those with frontal lobe dementia met DSM-III-R criteria for mild dementia and were in the early stages of the illness. Compared with patients with Alzheimer's disease, the group with frontal lobe dementia had significantly lower blood flow in the frontal lobes (dorsolateral and orbital), the anterior temporal cortex, and the basal ganglia. Within the frontal lobe dementia group, blood flow was significantly lower in the orbital than in the dorsal frontal cortex, and in the anterior temporal than in the dorsal temporal cortex. The present study shows the specificity of changes in regional cerebral blood flow in the diagnosis of different types of dementia, and supports the importance of orbitofrontal, anterior temporal, and basal ganglia dysfunction in the production of the psychiatric syndrome of frontal lobe dementia.
\end{abstract}

(F Neurol Neurosurg Psychiatry 1994;57:790-796)

Neary and coworkers described frontal lobe dementia as a degenerative condition characterised by changes in personality, breakdown in social conduct, disinhibition, impulsivity, unconcern, changes in eating conduct (hyperphagia), and stereotyped and perseverative behaviour. ${ }^{1}$ Disinhibited behaviour is often found among patients with neurological disorders such as closed head injuries and brain tumours, ${ }^{2}$ and may manifest itself as motor disinhibition (for example, hyperactivity, pressured speech, decreased need of sleep), intellectual disinhibition (for example, flight of ideas, grandiose delusions, paranoia), and instinctive disinhibition (for example, hypersexuality, hyperphagia). ${ }^{3}$ In some patients, disinhibited behaviour may be severe enough to fulfill DSM-III-R ${ }^{4}$ diagnostic criteria for mania, which require a distinct period of abnormally and persistently elevated, expansive, or irritable mood, and at least three of: inflated self esteem or grandiosity, decreased need for sleep, pressured speech, flight of ideas, distractibility, psychomotor agitation, and excessive involvement in pleasurable activities that have a high potential for painful results.

In a consecutive series of patients admitted to a psychiatric unit with the diagnosis of mania after a brain injury (stroke, tumour, or closed head injury) we found a high frequency of lesions involving the orbitofrontal cortex, anterior temporal cortex, head of the caudate, and thalamus. ${ }^{5}$ In a recent study that included a new series of seven consecutive patients with mania after brain injury, four had lesions involving the orbitofrontal or anterior temporal cortex, and the remaining three patients had single ischaemic lesions involving the right head of the caudate and the internal capsule. ${ }^{6}$ A PET study with ${ }^{18} \mathrm{~F}$-fluorodeoxyglucose $\left({ }^{18} \mathrm{FDG}\right)$ showed significant hypometabolic activity in the ipsilateral anterior temporal cortex in all three patients. Taken together, these findings suggest that disinhibited behaviour in patients with brain lesions may result from (direct or indirect) damage to the orbitofrontal and anterior temporal cortices. It is possible that similar changes may underlie the psychiatric disturbances of patients with frontal lobe dementia, as recent studies have shown significantly decreased blood flow in the frontal lobe of patients with a clinical diagnosis of frontal lobe dementia. ${ }^{7-10}$ Whether these changes in cerebral blood flow pertain to the disinhibition syndrome of frontal lobe dementia, however, rather than the cognitive impairment of this disorder, and whether there are specific areas within the frontal lobes that show blood flow deficits related to the behavioural change has not, to our knowledge, been empirically examined.

For the present study we included a series of patients with mild dementia who also met stringent criteria for disinhibited behaviour (the frontal lobe dementia group). To determine the severity, location, and specificity of blood flow deficits in frontal lobe dementia we included both an age matched control group and a series of age matched patients with a similar severity of dementia but no disinhibition who met the National Institute of Neurological and Communicative Disorder and Stroke-Alzheimer's Disease and Related Disorders Association ${ }^{11}$ criteria for probable Alzheimer's disease. Finally, to examine the importance of ventral structures in the production of disinhibited behaviour, we compared blood flow changes in orbital $v$ dorsal frontal areas, and anterior temporal $v$ dorsal temporal areas. 
Patients and methods PATIENTS

Frontal lobe dementia group

The frontal lobe dementia group consisted of eight patients who met the following inclusion criteria: (a) DSM-III-R diagnostic criteria for mild dementia, ${ }^{4}(b)$ a score $\geqslant 8$ or more on the Gustafson and Nilsson Pick scale, ${ }^{12}$ and a score $\leqslant 5$ on the Gustafson and Nilsson Alzheimer scale, ${ }^{12}(c)$ no CT evidence of focal brain lesions, $(d)$ normal results on routine laboratory tests, (e) a Hachinski ischaemic score $^{13}<5$, and $(f)$ no history of alcohol abuse or closed head injury.

\section{Alzheimer's disease group}

The Alzheimer's disease group consisted of eight patients who met the following inclusion criteria: (a) DSM-III-R diagnostic criteria of mild dementia, (b) a score on the Gustafson and Nilsson Alzheimer scale $>8$, and a score on the Gustafson and Nilsson Pick scale $<5$, (c) no CT evidence of focal brain lesions, (d) normal laboratory tests, (e) a Hachinski Ischaemic score $<5$, and $(f)$ no history of alcohol abuse or closed head injury.

\section{Control group}

The control group comprised four normal volunteers from our Institute and four people who complained of dizziness. All had a normal brain CT, a normal neurological evaluation, and no history of psychiatric disorders.

NEUROPSYCHIATRIC EXAMINATION

The neuropsychiatric evaluation consisted of the following:

\section{Mini-mental state examination (MMSE)}

The MMSE is an 11 item examination reliable and valid in assessing a limited range of cognitive functions. ${ }^{14}$

Gustafson and Nilsson rating scales for diagnosis of Alzheimer's disease and Pick's disease

This is a 16 item scale that scores the frequency of intellectual deficits (early amnesia, early disorientation, apraxia, aphasia, and agnosia), motor signs (increased muscular tension, myoclonic twitchings, and epileptic seizures of late onset), behavioural disorders (early loss of insight, Klüver-Bucy syndrome, early signs of disinhibition, irritability, and dysphoria), and the temporal progression of deficits (slow progression, and progressive reduction of spontaneity of speech). ${ }^{12}$ Gustafson and Nilsson showed that scores $<5$

Table 1 Demographic and neuropsychiatric findings

\begin{tabular}{lccc}
\hline & Controls & $\begin{array}{l}\text { Alzheimer's } \\
\text { group }\end{array}$ & $\begin{array}{c}\text { Frontal lobe } \\
\text { dementia }\end{array}$ \\
\hline No of patients & 8 & 8 & 8 \\
Age (mean y) & $67 \cdot 1(7 \cdot 5)$ & $70 \cdot 1(6 \cdot 8)$ & $68 \cdot 0(7 \cdot 4)$ \\
Sex (\% females) & 50 & 50 & 63 \\
Education (mean y) & $10 \cdot 5(4 \cdot 1)$ & $10 \cdot 2(5 \cdot 3)$ & $11 \cdot 1(4 \cdot 7)$ \\
Duration of illness (mean y) & & $2 \cdot 4(1 \cdot 8)$ & $4 \cdot 1(3 \cdot 3)$ \\
Mini-mental state exam (mean scores) & $29 \cdot 5(2 \cdot 2)$ & $21 \cdot 6(5 \cdot 3)$ & $21 \cdot 5(4 \cdot 3)$ \\
Gustafson-Nilsson Alzheimer scale & & $8 \cdot 1(0 \cdot 3)$ & $3 \cdot 2(1 \cdot 2)$ \\
Gustafson-Nilsson Pick scale & & $2 \cdot 3(1 \cdot 0)$ & $9 \cdot 2(0 \cdot 4)$ \\
\hline
\end{tabular}

Figures in parentheses are SDs. on the Alzheimer's scale and $>5$ on the Pick's scale had a sensitivity and specificity of $100 \%$ for the diagnosis of pathologically established Pick's disease. On the other hand, scores $<5$ on the Pick's scale and $>8$ on the Alzheimer's scale had a sensitivity and specificity of $100 \%$ for the diagnosis of pathologically established Alzheimer's disease. Thus in the present study patients included in the Alzheimer's group had a score $\leqslant 5$ on the Pick's scale and a score $>8$ on the Alzheimer's scale, whereas patients included in the frontal lobe dementia group had a score $\leqslant 5$ on the Alzheimer's scale and $>8$ on the Pick's scale (we decided to be more stringent on the Pick's scale score for the frontal lobe dementia group so as to maximise the chance of including patients with severe disinhibition) (table 1).

NEUROPSYCHOLOGICAL EXAMINATION

Patients with Alzheimer's disease and patients with frontal lobe dementia had a comprehensive neuropsychological evaluation that was carried out within two weeks of the SPECT study and included the following tasks:

Wechsler adult intelligence scale-revised (WAIS-R)

The WAIS-R is a measure of general intellectual ability.

\section{Wisconsin card sorting test}

This test measures the ability to develop new concepts and shift sets, and also requires the subject to suppress a previously correct response and produce a new one. ${ }^{16}$

\section{Controlled word association test}

This test examines access to semantic information with time constraint. ${ }^{17}$

\section{Trail making test}

This test examines visual, conceptual, and visuomotor tracking. ${ }^{18}$

\section{Digit span}

This test examines auditory attention. ${ }^{19}$

\section{Buschke selective reminding test}

This test measures verbal learning and memory during a multiple trial list learning task. ${ }^{20}$

\section{Benton visual retention test}

This test assesses visual perception and visual memory. ${ }^{21}$

\section{Token test}

This test examines verbal comprehension of sentences of increasing complexity. ${ }^{22}$

Boston naming test

This test examines the ability to name pictured objects. ${ }^{23}$

\section{Raven's progressive matrices}

This test assesses reasoning in the visual modality. ${ }^{24}$

Apraxia subtest of the western aphasia battery This test examines the presence of ideomotor apraxia. ${ }^{25}$ 
Block design

This test examines the presence of constructional apraxia. ${ }^{15}$

\section{Similarities}

This test provides a measure of abstract reasoning. ${ }^{15}$

\section{SPECT EXAMINATION}

After written consent was obtained from patients and controls, a SPECT study was carried out. This study was approved by the ethics committee of the Raúl Carrea Institute of Neurological Research. We used ${ }^{99 \mathrm{~m}} \mathrm{Tc}-$ HMPAO (25 mCi) (Ceretec, Exametazime, Amersham International), which was injected intravenously in an antecubital vein. Patients or control subjects sat with their eyes open and ears unplugged in a quiet room with dim lights. Fifteen minutes after the injection they were positioned supine and with the orbitomeatal line positioned vertically, centred in the field of view. The alignment was carried out with vertical and horizontal laser beams, and the head was held still by an ad hoc head holder. SPECT was carried out with a General Electric $400 \mathrm{AC} / \mathrm{T}$ rotating gamma camera attached to a Starcam 3200 computer.

We used a high resolution collimator and a $64 \times 64$ matrix. There were 64 images obtained over $360^{\circ}$, with a 30 second acquisition time and a zoom of $1 \cdot 6$. Processing was carried out with a Butterworth filtering, a critical frequency of 0.44 , and a slice width of 1 pixel. Following the procedure of Burns et al ${ }^{26}$ square regions of interest of $4 \times 4$ pixels were used to obtain activity ratios in axial slices, taking the cerebellum as reference. Three measurements were carried out for each of the following cortical areas: frontal dorsolateral, frontal orbital, anterior temporal, temporal dorsal, and parietal. These measurements were averaged for each cortical region on the right and left hemispheres. Regions of interest were also placed in the basal ganglia, thalamus, and cerebellum. To determine the activity ratio (brain region/cerebellum), the counts per region of interest of each cortical area were divided by the counts per region of

Table 2 Neuropsychological findings

\begin{tabular}{lcc}
\hline Neuropsychological task & $\begin{array}{l}\text { Alzheimer's } \\
\text { group }\end{array}$ & $\begin{array}{c}\text { Frontal lobe } \\
\text { dementia }\end{array}$ \\
\hline Buschke SRT-long term retrieval & $23 \cdot 0(13 \cdot 0)$ & $12 \cdot 2(11 \cdot 0)$ \\
Buschke SRT_short term retrieval & $28 \cdot 7(7 \cdot 2)$ & $28 \cdot 5(9 \cdot 6)$ \\
Buschke SRT—-long term storage & $30 \cdot 5(15 \cdot 0)$ & $17 \cdot 2(15 \cdot 2)$ \\
Benton visual retention test & $5 \cdot 7(3 \cdot 4)$ & $5 \cdot 8(1 \cdot 7)$ \\
Token test & $19 \cdot 2(4 \cdot 3)$ & $21 \cdot 0(2 \cdot 7)$ \\
Boston naming test & $14 \cdot 5(2 \cdot 9)$ & $13 \cdot 6(5 \cdot 7)$ \\
Wisconsin card sorting test-categories & $3 \cdot 1(2 \cdot 4)$ & $2 \cdot 3(1 \cdot 5)$ \\
Wisconsin card sorting test-perseverations & $15 \cdot 8(14 \cdot 1)$ & $19 \cdot 7(9 \cdot 8)$ \\
Trial making test part A-time (s) & $85 \cdot 5(55 \cdot 0)$ & $70 \cdot 1(31 \cdot 0)$ \\
Trial making test part A-number of errors & $0 \cdot 4(1 \cdot 1)$ & $0 \cdot 2(0 \cdot 7)$ \\
Trial making test part B-time (s) & $307(189)$ & $259(172)$ \\
Trial making test part B-number of errors & $4 \cdot 1(4 \cdot 1)$ & $3 \cdot 2(4 \cdot 3)$ \\
Controlled word association test & $31 \cdot 2(5 \cdot 5)$ & $25 \cdot 8(8 \cdot 0)$ \\
Raven's progressive matrices-percentile & $45 \cdot 5(40 \cdot 9)$ & $53 \cdot 1(26 \cdot 5)$ \\
Digit span-forward & $5 \cdot 1(0 \cdot 6)$ & $5 \cdot 7(0 \cdot 8)$ \\
Digit span-backward & $2 \cdot 8(1 \cdot 4)$ & $3 \cdot 8(1 \cdot 1)$ \\
Ideomotor-apraxia & $58 \cdot 4(2 \cdot 0)$ & $56 \cdot 8(6 \cdot 8)$ \\
Block design & $3 \cdot 7(2 \cdot 6)$ & $5 \cdot 1(1 \cdot 4)$ \\
Analogies & $10 \cdot 7(4 \cdot 8)$ & $8 \cdot 1(5 \cdot 4)$ \\
Wechsler adult intelligence scale-total IQ & $83 \cdot 6(14 \cdot 6)$ & $87 \cdot 0(12 \cdot 8)$ \\
\hline
\end{tabular}

Figures in parentheses are SDs. interest found in the cerebellar hemisphere with the highest average count. This ratio was used as a measure of regional cerebral blood flow. ${ }^{10}$ All SPECT measurements were carried out by a neuroradiologist blind to the clinical data, and showed high inter-rater and intrarater reliability $(r=0.89$ and 0.94 , $\mathrm{df}=139, \mathrm{p}=0.001)$.

\section{STATISTICAL ANALYSIS}

Statistical analysis was carried out from means and SDs, analysis of variance with repeated measures (ANOVA), and a Tukey post hoc test. All $\mathrm{p}$ values are two tailed.

\section{Results}

DEMOGRAPHIC FINDINGS (TABLE 1)

No significant differences were found between Alzheimer's, frontal lobe dementia, and control groups in age, education, and sex distribution. Alzheimer's and frontal lobe dementia groups had a similar duration of illness.

NEUROPSYCHOLOGICAL FINDINGS (TABLE 2)

There were no significant differences in minimental state exam scores, WAIS-R scores, verbal and visual memory, language, apraxia, attention, and frontal lobe related tasks between patients with frontal lobe dementia or Alzheimer's disease.

The disinhibition syndrome of the frontal lobe dementia group was characterised by motor hyperactivity in $75 \%$ of the patients, logorrhea in $63 \%$, confabulation in $100 \%$, irritability in $75 \%$, euphoria in $88 \%$, sexual disinhibition in $63 \%$, verbal disinhibition in $63 \%$, and orality in $50 \%$.

\section{SPECT-FINDINGS (TABLE 3)}

A three way ANOVA with repeated measures for cerebral blood flow (factor 1: group (Alzheimer's disease $v$ frontal lobe dementia $v$ controls), factor 2: region (frontal dorsal, frontal orbital, temporal dorsal, anterior temporal, parietal, basal ganglia, and thalamus), and factor 3: side (left $v$ right)) showed a significant group effect $(F(2,21)=15 \cdot 3, p=$ 0.00007 ); patients with frontal lobe dementia had a significantly lower global cerebral blood flow than patients with Alzheimer's disease and controls $(p=0.01$, and $p=0.0001$, respectively). There was also a significant group $\times$ region interaction $(F(12,126)=$ $3.74, \mathrm{p}=0.00007)$; on individual comparisons patients with Alzheimer's disease had a significantly lower temporal dorsal blood flow than controls $(p=0.003)$, whereas patients with frontal lobe dementia showed a significantly lower blood flow in frontal (dorsal $p=$ 0.00005 , orbital $p=0.00004$ ), temporal (dorsal $p=0.0006$, anterior $p=0.0004$ ), and basal ganglia regions $(p=0.0007)$ than controls. Finally, the frontal lobe dementia group showed significantly lower blood flow than patients with Alzheimer's disease in the orbitofrontal cortex $(p=0.00004)$, frontal dorsolateral cortex $(p=0.015)$, anterior temporal cortex $(p=0.016)$, and basal ganglia $(p=0.010)$. No significant between group 
Table 3 Measurement of regional cerebral blood flow

\begin{tabular}{llll}
\hline Brain area & Control group & Alzheimer's group & $\begin{array}{l}\text { Frontal lobe } \\
\text { dementia group }\end{array}$ \\
\hline Frontal orbital & $81 \cdot 2(6 \cdot 9)$ & $76 \cdot 1(13 \cdot 7)$ & $58 \cdot 2(11 \cdot 1)$ \\
Frontal dorsolateral & $85 \cdot 8(4 \cdot 4)$ & $81 \cdot 8(7 \cdot 7)$ & $71 \cdot 4(9 \cdot 2)$ \\
Temporal anterior & $91 \cdot 7(4 \cdot 4)$ & $85 \cdot 3(5 \cdot 5)$ & $74 \cdot 9(5 \cdot 8)$ \\
Temporal dorsal & $90 \cdot 9(4 \cdot 5)$ & $79 \cdot 4(5 \cdot 2)$ & $78 \cdot 5(4 \cdot 9)$ \\
Parietal & $91 \cdot 7(6 \cdot 7)$ & $82 \cdot 8(7 \cdot 0)$ & $82 \cdot 4(7 \cdot 3)$ \\
Basal ganglia & $93 \cdot 0(6 \cdot 6)$ & $91 \cdot 3(8 \cdot 5)$ & $80 \cdot 6(8 \cdot 9)$ \\
Thalamus & $86 \cdot 2(6 \cdot 9)$ & $84 \cdot 8(7 \cdot 6)$ & $79 \cdot 4(5 \cdot 4)$ \\
\hline
\end{tabular}

Figures in parentheses are SDs.

differences were found in the remaining brain areas.

To test the relative importance of orbital $v$ dorsal frontal blood flow changes in the production of disinhibition, we carried out a three way ANOVA with repeated measures (factor 1: group (Alzheimer's $v$ frontal lobe dementia), factor 2: region (frontal dorsal $v$ frontal orbital), and factor 3: side (left $v$ right)). We found a significant group effect (patients with frontal lobe dementia had an overall significantly lower blood flow in the frontal lobes) $(F(1,14)=8.95, p=0.009)$, and a nearly significant group $\times$ region interaction $(F(1,14)=3.98, p=0.06)$. Patients with frontal lobe dementia had significantly lower blood flow in the frontal orbital cortex than in the frontal dorsal cortex $(p=0.0002)$ (fig 1). On the other hand, there were no significant differences between these cortical regions in the Alzheimer's group (fig 2).

To test the relative importance of anterior $v$ dorsal temporal blood flow changes in the production of disinhibition we carried out a three way ANOVA with repeated measures, in which the region factor was anterior $v$ dorsal temporal blood flow. There was a significant group effect (patients with frontal lobe dementia had an overall significantly lower blood flow in the temporal lobe; $F(1,14)=$ $8 \cdot 68, \mathrm{p}=0.01$ ), as well as a significant group $\times$ region interaction $(\mathrm{F}(1,14)=11 \cdot 7, \mathrm{p}=$ 0.004). Whereas patients with Alzheimer's and frontal lobe dementia had a comparable blood flow in the dorsal temporal cortex

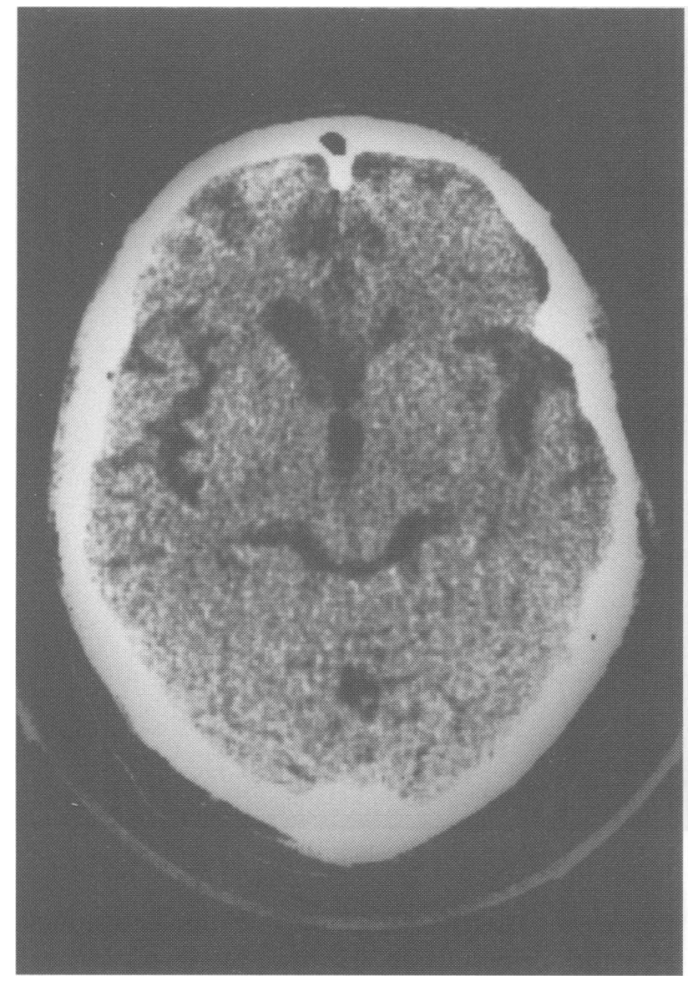

Figure 1 Top left: $C T$ scan of a patient with frontal lobe dementia showing mild prominence of cortical sulci. Top right: SPECT scan showing frontal lobe blood flow deficits in the axial, saggital, and coronal planes. Bottom right: three dimensional rendering of the SPECT study showing the frontal lobe perfusion defect but no posterior brain blood flow changes.

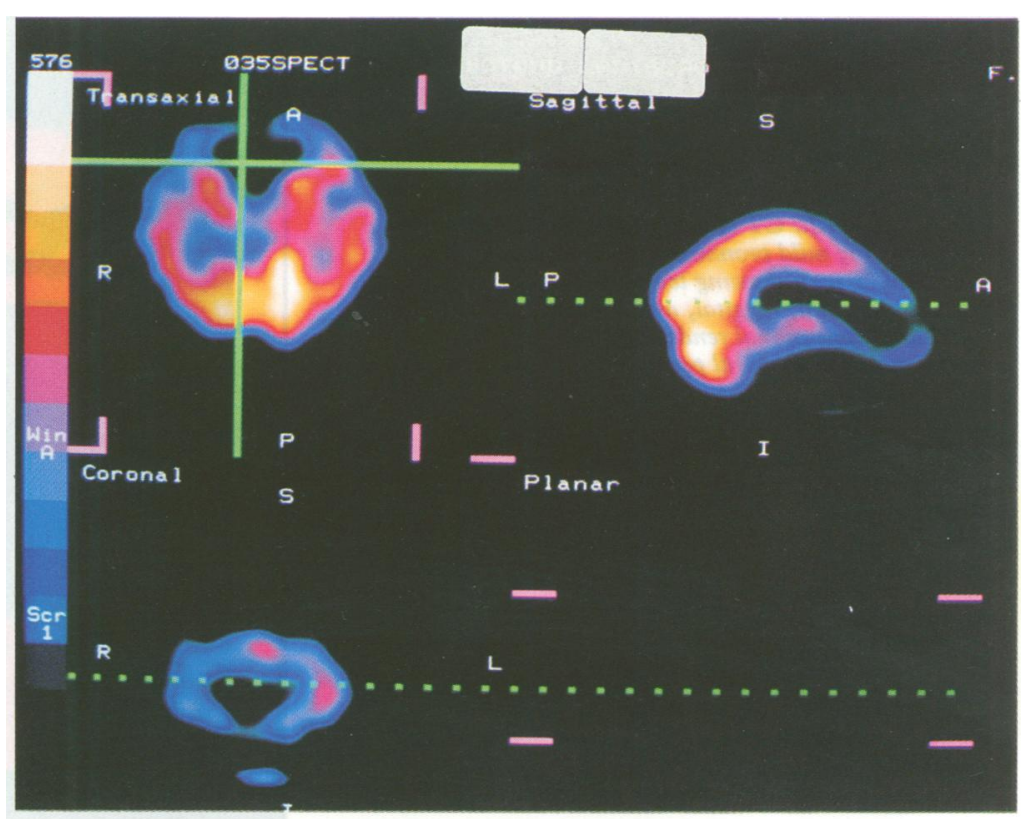




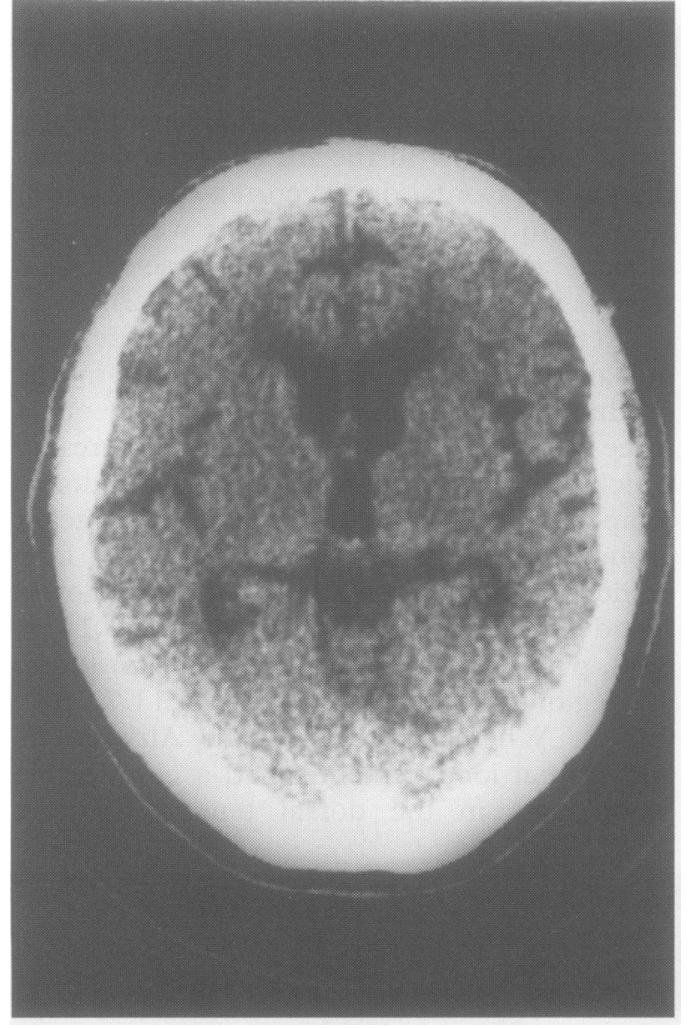

Figure 2 Top left: CT of a patient with Alzheimer's disease showing mild prominence of cortical sulci. Top right: SPECT scan showing temporo-parietal blood flow deficits in the axial, saggital, and coronal planes. Bottom right: Three dimensional rendering of the SPECT study showing posterior temporal perfusion deficits but no frontal lobe changes.
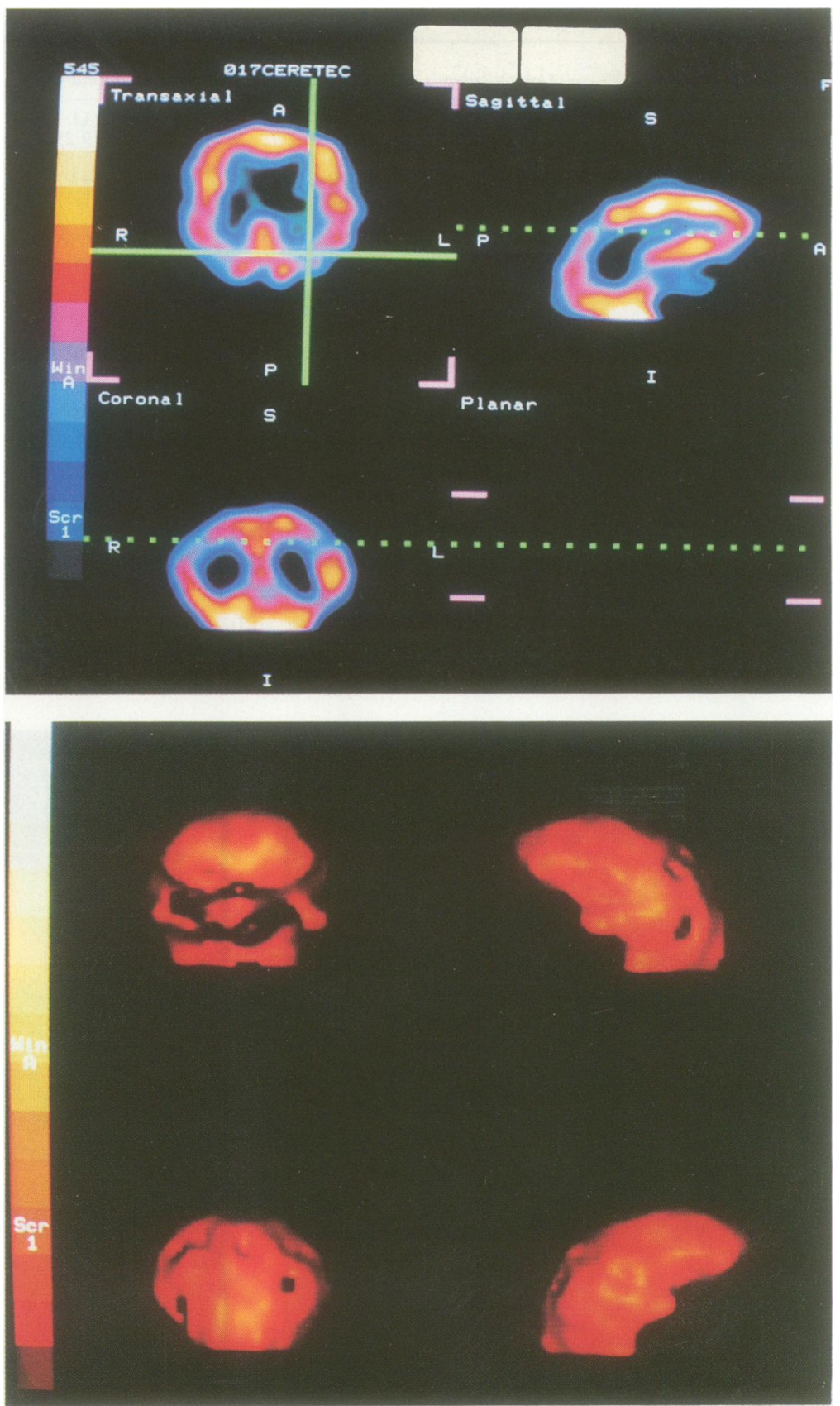

$(p=0.63)$, patients with frontal lobe dementia had a significantly lower blood flow in the anterior temporal cortex $(\mathrm{p}=0.0001)$.

\section{Discussion}

The main finding of the present study was that patients with mild dementia and a disinhibition syndrome had significant blood flow deficits in specific brain areas. When compared with controls, these changes primarily involved the frontal and temporal lobes and the basal ganglia. When compared with patients with mild dementia but no disinhibited behaviour (the Alzheimer's group), patients with frontal lobe dementia showed significant blood flow deficits involving primarily the orbital frontal cortex and the anterior temporal cortex.

Before further discussion, some limitations of our study should be pointed out. One important methodological problem is the lack of valid criteria for the diagnosis of frontal lobe dementia or Pick's disease. We tried to deal with this limitation by using the Gustafson and Nilsson scale for Alzheimer and Pick's disease. ${ }^{12}$ This is a clinical scale that rates symptoms of intellectual decline, disinhibition, and motor signs, and was found to be valid for the diagnosis of pathologically established Alzheimer's and Pick's diseases. Moreover, to include patients with the highest probability of having Alzheimer's or Pick's disease, we used a high cut off score on this scale. Although we used the terms frontal lobe dementia or Pick's disease for the same group of patients, some but not all patients with frontal lobe dementia have been reported to have Pick's disease, ${ }^{7}$ and not all patients with Pick's disease have the clinical syndrome of frontal lobe dementia. ${ }^{27}$ Regardless of the ultimate cause, what we have tried to examine 
was disinhibition, a behaviour tightly linked to both frontal lobe dementia and Pick's disease. Thus we are showing significant differences in cerebral blood flow between mildly demented patients with and without disinhibited behaviour, regardless of the final neuropathological diagnosis. Another limitation is our uncertainty about the stability of these findings. We did not rescan the patients at follow up or in a different setting (for example, conducting an attention task). Thus it is possible that our present findings are only valid for one stage of the disorder and only in the environment where the patients were tested.

Neary et $a l^{1}$ described a group of patients with mild dementia and striking behavioural changes, such as unconcern, inappropriate jocularity, distractibility, loss of social awareness, loss of emotional empathy, hyperorality, and obsessionality. They termed this entity frontal lobe dementia, emphasising the topography of pathology rather than its pathogenesis, and speculated that frontal lobe dementia may be one type of Pick's disease. The same authors have recently reported four patients with frontal lobe dementia and motor neuron disease, all of whom showed important personality changes such as impulsivity, unconcern, breakdown in social conduct, changes in eating habits, and stereotyped and perseverative behaviour. ${ }^{7}$ Neuropathological examination showed extensive atrophy involving the frontal lobes, anterior temporal cortex, insula, and the anterior cingulate. Microscopical examination showed neuropathological changes consistent with progressive subcortical gliosis.

Only a few studies have empirically examined the presence of changes in cerebral blood flow in patients with frontal lobe dementia. Neary et al carried out SPECT in patients with either Alzheimer's disease, frontal lobe dementia, or progressive supranuclear palsy. ${ }^{28}$ Whereas they found blood flow deficits in posterior brain areas in patients with Alzheimer's disease, patients with either frontal lobe dementia, or progressive supranuclear palsy showed significant blood flow deficits in anterior brain areas. Miller et al have recently reported frontal and anterior temporal hypoperfusion as well as relative sparing of parietal and occipital blood flow in patients with frontal lobe dementia compared with normal controls. ${ }^{8}$ Although Miller et al obtained quantitative information, the diagnosis of frontal lobe dementia was only established after patients showed both deficits on frontal lobe related cognitive tasks and selective hypoperfusion of the frontal lobes.

Studies with PET in patients with either frontal lobe dementia or Pick's disease showed similar findings. Kumar et $a l^{29}$ have recently reported three patients with dementia and frontal lobe behavioural changes such as sexual disinhibition, irritability, laughing bursts, and loud speech that showed metabolic deficits bilaterally in the orbitofrontal, anterior cingulate, and temporal lobes, with relative sparing of the parietal lobes. Significant frontal lobe hypometabolism was also reported in patients with Alzheimer's disease and agitation, inappropriate behaviour, and personality changes, ${ }^{30} 31$ and patients with histologically established Pick's disease..$^{32} 33$

In the present study we included patients with either Alzheimer's disease or frontal lobe dementia with mild dementia as well as an age matched control group. All our patients with frontal lobe dementia met stringent criteria for disinhibition, and neither the presence of deficits in frontal lobe related tasks nor the pattern of SPECT abnormalities were considered as inclusion criteria. Our study fully replicates that of Neary $e t a l^{28}$ in finding frontal and anterior temporal blood flow deficits in patients with frontal lobe dementia.

The question that now arises is how these findings may be explained? Perhaps the most fundamental issue is whether these decreases in regional cerebral blood flow are a cause of the disinhibition, a consequence, or are related to a third independent factor. Although we cannot fully answer this question, the fact that the decreased cerebral blood flow occurred in areas associated with disinhibited behaviour in patients with structural brain injury ${ }^{5}$ suggests that dysfunction of these brain areas (as shown by diminished cerebral blood flow) may lead to these behavioural changes.

Whereas the disinhibited behaviour of frontal lobe dementia may be secondary to orbitofrontal and anterior temporal pathology, other dementing disorders, such as progressive subcortical gliosis, Lewy body disease, Pick's disease, and even Alzheimer's disease, may show disinhibited behaviour provided there is concomitant dysfunction of these cortical regions. In 1941, Kennard et al showed that lesions of the orbitofrontal cortex that extended into the caudate nucleus invariably produced locomotor hyperactivity. ${ }^{34}$ Kling and Stelkis ${ }^{35}$ reported that posterior orbitofrontal resections in primates produced important behavioural changes, such as hyperorality, coprophagia, pacing and circling, irritability, inappropriate facial expressions, and vocalisations, which resulted in "social disintegration". These changes were not present in primates with frontal dorsolateral resections. Based on these findings they suggested that bilateral lesions of the orbitofrontal cortex or the anterior temporal lobe critically disrupt behaviours of social bonding.

In conclusion, we have replicated previous findings of frontal lobe hypoperfusion in patients with dementia and disinhibited behaviour. The largest blood flow changes were found in the orbitofrontal and anterior temporal areas compared with dorsal frontal and dorsal temporal regions. These findings suggest that disinhibited behaviour in mildly demented patients may result from dorsal neocortical and limbic release from cortical ventral control. As beautifully expressed by Bianchi in $1922^{36}$ "the higher sentiments ...., above all, ... disappear after mutilation of the frontal lobes, whilst the primitive emotions, ... especially . . . irrational, . . . remain, sometimes even intensive ..." 
This work was partially supported by grants from the Instituto Di Tella and the Sandoz Foundation. We thank Dr Juan Goldar for his many valuable suggestions.

1 Neary D, Snowden JS, Northen B, Goulding PJ. Dementia of frontal lobe type. $\mathcal{F}$ Neurol Neurosurg Psychiatry 1988; 51:353-61

2 Cummings $\mathrm{JL}$. Organic psychoses: delusional disorders and secondary mania. Psychiatr Clin North Am 1986;9: 293-311.

3 Starkstein SE, Robinson RG. The role of the frontal lobes in affective disorder following stroke. In: Levin $\mathrm{H}$, ed. Neurobehavioral consequences of closed head injuries. New York: Oxford University Press, 1991:288-303.

4 American Psychiatric Association. Diagnostic and statistical manual of mental disorders-revised. Washington, DC American Psychiatric Press, 1987.

5 Starkstein SE, Boston JD, Robinson RG. Mechanisms of mania after brain injury: 12 case reports and review of mania after brain injury: 12 case reports and re

6 Starkstein SE, Mayberg HS, Berthier ML, et al. Mania after brain injury: neuroradiological and metabolic findafter brain injury: neuroradiolog
ings. Ann Neurol 1990;27:652-9.

7 Neary D, Snowden JS, Mann DMA, Northen B, Goulding PJ, Macdermott N. Frontal lobe dementia and motor neuron disease. $\mathcal{f}$ Neurol Neurosurg Psychiatry 1990;53 23-32.

8 Miller BL, Cummings JL, Villanueva-Meyer J, et al. Frontal lobe degeneration: clinical, neuropsychological, and SPECT characteristics. Neurology 1991;41 1374-82.

9 Risberg J. Frontal lobe degeneration of non-Alzheimer type. III. Regional cerebral blood flow. Arch Gerontol Geriatr 1987;6:225-33.

10 Jagust WJ, Reed BR, Seab JP, Kramer JH, Budinger TF. Clinical-physiologic correlates of Alzheimer's disease and frontal lobe dementia. Am $\mathcal{F}$ Physiol Imaging and frontal 1980 .

11 McKhann G, Drachman D, Folstein MF, Katzman R, Price D, Stadlan EM. Clinical diagnosis of Alzheimer's disease: report of the NINCDS-ADRDA Work Group under the auspices of Department of Health and Human Services Task Force on Alzheimer's Disease. Neurology 1984;34:939-44.

12 Gustafson I, Nilsson L. Differential diagnosis of presenile dementia on clinical grounds. Acta Psychol Scand 1982;65:194-209.

13 Hachinski VC, Iliff LD, Zilhka L, et al. Cerebral blood flow in dementia. Arch Neurol 1975;40:97-103.

14 Folstein MF, Folstein SE, McHugh PR. Mini-Mental state: a practical method for grading the cognitive state
of patients for the clinician. $\mathcal{F}$ Psychiatr Res 1975; 12:189-98.

15 Wechsler D. Wechsler adult intelligence scale-revised. New York: The Psychological Corporation, 1981.

16 Nelson HE. A modified card sorting test sensitive to frontal lobe defects. Cortex 1976;12:313-24.
17 Benton AL. Differential behavioral effects in frontal lobe disease. Neuropsychologia 1968;6:53-60.

18 Reitan RM. Validity of the trail making test as an indicator of organic brain damage. Percept Mot Skills 1958;8: 271-6.

19 Wechsler D. A standardized memory scale for clinical use. f Psychol 1945;19:87-95.

20 Buschke H, Fuld PA. Evaluating storage, retention, and retrieval in disordered memory and learning. Neurology

21 Benton AL. The revised visual retention test, 4 th ed. New York: The Psychological Corporation, 1974

22 De Renzi E, Faglioni P. Development of a shortened version of the Token Test. Cortex 1978;14:41-9.

23 Kaplan EF, Goodglass H, Weintraub S. The Boston naming test. Philadelphia: Lea and Febiger, 1983.

24 Raven JC, Court JH, Raven J. Manual for Raven's progressive matrices and vocabulary scales. London: $\mathrm{H} \mathrm{K}$ Lewis and Co, Ltd, 1986.

25 Kertesz A. Western aphasia battery. New York: Grune and Stratton, 1982.

26 Burns A, Philpot MP, Costa DC, Ell PJ, Levy R. The investigation of Alzheimer's disease with single photon emission tomography. $₹$ Neurol Neurosurg Psychiatry $1989 ; 12: 248-53$.

27 Cummings JL, Duchen LW. Klüver-Bucy syndrome in Pick disease: clinical and pathological correlations. Neurology 1981;31:1415-22.

28 Neary D, Snowden J, Shields R, et al. Single photon emission tomography using $99 \mathrm{mTc}-\mathrm{HM}-\mathrm{PAO}$ in the investigation of dementia. F Neurol Neurosurg Psychiatry 1987; 50:1101-9.

29 Kumar A, Schapiro MB, Haxby JV, Grady CL, Friedland RP. Cerebral metabolic and cognitive studies in dementia with frontal lobe behavioral features. 7 Psychiatr Res 1990;24:97-109.

30 Chase TM. Cortical glucose utilization patterns in primary degenerative dementias of the anterior and posterior degenerative dementias of the anterior and

31 Grady CI Haxy JV, Schapiro MB, et al Subgroups in dementia of the Alzheimer type identified using positron dementia of the Alzheimer type identified using positron 1990;2:373-84.

32 Kamo H, McGeer PL, Harrop R, McGeer EG, Calne DB, Martin WRW, Pate BD. Positron emission tomography and histopathology in Pick's disease. Neurology 1987; 37:439-45.

33 Brion S, Mikol J, Baron JC, Plas J, Guerin R, Bessac JF. Neuroimaging in Pick's disease with histological data. $\mathcal{f}$ Neurol 1988;235(suppl 1):15-22.

34 Kennard MA, Spencer S, Fountain G. Lesions of the orbital cortex of the frontal lobe which invade the caudate nucleus produce locomotor hyperactivity. F Neurophysiol 1941;4:512-24.

35 Kling A, Steklis HD. A neural substrate for affiliative behavior in nonhuman primates. Brain Behav Evol 1976;13:216-38.

36 Bianchi L. The mechanism of the brain and the function of the frontal lobes. New York: William Wood, 1922. 